\title{
Editorial
}

\section{The Evolving World of Healthcare-Associated Bloodstream Infection Surveillance and Prevention: Is Your System as Good as You Think?}

\author{
William R. Jarvis, MD
}

\begin{abstract}
Bloodstream infections (BSIs) are one of the most common healthcare-associated infections and a leading cause of morbidity and mortality. From 1980 to 1990 , the incidence of BSIs in U.S. hospitals was estimated to have increased by nearly $70 \% .{ }^{1}$ In hospitalized patients, BSIs are estimated to account for approximately $10 \%$ of all healthcare-associated infections and to have an attributable mortality rate of approximately $15 \%$, making them the eighth leading cause of death in the United States. ${ }^{2}$ At most hospitals, surveillance for BSIs is thought to be very complete. After all, most BSIs are diagnosed via blood culture and cultures are obtained from most symptomatic patients. A validation study conducted in National Nosocomial Infections Surveillance (NNIS) system hospitals showed that NNIS system surveillance for BSIs had a positive predictive value of $87 \%$, a sensitivity of $85 \%$, and a specificity of $98.3 \%{ }^{3}$

However, as illustrated by the article by Smith et al. ${ }^{4}$ in this issue of Infection Control and Hospital Epidemiology, many aspects of our healthcare system and healthcare delivery processes have changed in the past decade, complicating our efforts to conduct surveillance, calculate BSI rates, and implement BSI (and other infection) prevention interventions. These aspects include enormous changes in the way in which and where we provide health care, reductions in clinical personnel, increased use of intravascular catheters (particularly central venous catheters [CVCs]) for a wider variety of reasons and in more clinically diverse populations, reductions in or outsourcing of clinical microbiology services, and failure to integrate our healthcare setting clinical information systems. All of these lead to the potential to have much less robust or complete BSI surveillance systems and thus much less effective BSI prevention and control programs than we think.
\end{abstract}

Let's examine how these changes influence our BSI prevention programs. First, let's examine what has happened to our healthcare delivery system. During the past two decades, there has been a dramatic shift in the delivery of health care from inpatient to outpatient settings. In 1996, nearly $8,000,000$ individuals in the United States received medical care in their home; approximately $10 \%$ or an estimated 774,113 had at least one indwelling medical devicemost of these being intravascular catheters. ${ }^{5}$ In that same year, approximately $750,000,000$ individuals received care in a physician's office, $75,000,000$ visited a hospital emergency department, and 50,000,000 received care at a hospital outpatient clinic. ${ }^{6}$ In 1999 , approximately $82.5 \%$ of the entire U.S. population had more than one visit for receipt of health care to a physician's office, an emergency department, or a clinic or received care at their home. ${ }^{7}$ In the "outbreak of BSIs" investigated by Smith et al., this complex system of healthcare delivery complicated the infection control program's surveillance for BSIs and the "outbreak" investigation. The initial perception of an increase in BSIs was based on clinical perception and numerator data. This occurred because denominator data to calculate BSI rates were not readily available, as the patients were being seen in the outpatient clinic and there was no computer database of who (much less what their service or diagnosis was) visited the outpatient clinic, whether those visiting the clinic had a CVC, or whether the catheter was manipulated in the clinic. Initially, because all of the patients had the onset of symptoms as outpatients and were included in a home infusion therapy program, the BSIs were thought to be secondary to home infusion therapy. The majority of patients with BSIs had periodically received home infusion therapy (provided by several companies that did not provide BSI 
numerator, denominator, or BSI rate data); however, most of the patients were not receiving home infusion therapy in the days or weeks immediately preceding the onset of their BSI. Thus, determining where (inpatient, clinic, emergency department, or physician's office) each patient is receiving medical care and where his or her intravascular catheter is manipulated is both important and increasingly more difficult. This is critical if infection control personnel are to identify where lapses in aseptic technique or other practices, which could lead to an adverse event, are occurring so that education programs can be targeted. On further investigation, Smith et al. found that most of the home infusion therapy companies provided supplies, but not health care, and family members were instructed in aseptic technique and how to provide the care at the time of the patient's initial admission and oncologic diagnosis. Appropriate education on infusion therapy and infection control techniques for family members who will be providing care is essential, if complications are to be avoided; language and level of training materials have been shown to be important in preventing BSIs. ${ }^{8}$ Studies are needed on whether teaching family members at the time of the initial diagnosis (when there is considerable family anxiety and turmoil) is sufficient or whether periodic additional education is needed.

Second, let's examine the role of clinical personnel staffing and BSIs. Although not evaluated in this investigation, across the United States there are reports of downsizing of clinical care staff and nursing shortages. Throughout the world, shortages of clinical personnel often are the rule, not the exception. Both inadequate numbers of personnel and inadequately trained personnel increase the risk of BSIs. Fridkin et al. and Archibald et al. showed that in inpatient settings, reductions in intensive care unit nurse-to-patient ratios (fewer nurses for more patients) were associated with increased BSI rates., ${ }^{9,10}$ Robert et al. showed that not only is the nurse-to-patient ratio important, but the training of those personnel (and their familiarity with unit practices) also influences BSI risk. ${ }^{11}$ Few, if any, studies have evaluated nurse staffing or medical device insertion or manipulation practices in outpatient settings or the infection control practices of these staff, who are increasingly performing more complex and invasive diagnostic and therapeutic procedures. Interestingly, Tokars et al. showed that one of the major risk factors for BSI in patients receiving home infusion therapy was a recent visit for medical care (and presumed CVC manipulation) to a private physician's office. ${ }^{12}$ As health care is increasingly provided in these outpatient settings, infection control personnel will need to ensure that infection control education programs reach these healthcare personnel and that active surveillance systems for detection of BSIs reach these areas of the expanding healthcare environment.

The third factor complicating BSI surveillance and prevention is the increasing prevalence of CVCs. The dramatic increase in healthcare-associated BSI rates has paralleled the increasing use of CVCs in a wide variety of patient populations. Not only are there an increasing variety of types of CVCs-tunneled, nontunneled, midline, central, peripherally inserted, antiseptic, and antimicrobial-coated-but the healthcare system location of their insertion and the diversity of the population of patients in whom they are placed have rapidly grown during the past one to two decades. Today, CVCs routinely are inserted in operating rooms, patient wards, intensive care units, radiology suites, private physician offices, and outpatient settings. Are we sure that all of the personnel inserting these catheters are properly instructed and are aware of and comply with current recommendations? ${ }^{13}$ We know that these recommendations, if put into practice, can reduce BSIs and that education of our clinicians is effective in reducing BSIs. As outpatient and home therapy have emerged, many patients receiving care in these settings have CVCs. This includes hematology-oncology patients, transplant recipients, and patients infected with human immunodeficiency virus. Many of these patients are at increased risk of infection with common or emerging pathogens, yet often they are not properly instructed in how to care for their CVCs or protect them from common exposures, such as tap water during bathing or outdoor activities. Numerous instances and outbreaks of gram-negative bacterial or nontuberculous mycobacterial BSIs occur each year (especially in the summer) in patients with long-term indwelling intravascular catheters from such exposures. As reimbursement for home therapy has declined, more and more home care companies are having to decrease the nurse-to-patient ratios and depend on family members to provide the infusion therapy. Additional educational activities may be needed to ensure that providers and recipients are aware of the latest guidelines for prevention of BSIs, and periodic observational studies may be needed to ensure that policies are actually being practiced. Who has the responsibility and the time to undertake these activities?

The fourth factor influencing BSI detection is the changing world of clinical microbiology. The Study of the Efficacy of Nosocomial Infection Control (SENIC) program showed that the frequency of the use of diagnostic tests influences infection rates. ${ }^{14}$ Until recently, many might have argued that, in the United States, blood cultures are ordered too frequently (ie, for too many patients and too many cultures for any one patient); thus, the likelihood that an episode of BSI would be missed would be low. However, current changes in clinical microbiology threaten that premise. At many facilities, the clinical microbiology laboratory, which was in the hospital, has been closed and specimens are now outsourced to a contract laboratory, across the city or across the country. The situation is further complicated for the population of patients with long-term indwelling $\mathrm{CVCs}$, as they may develop clinical signs and symptoms of BSI and be evaluated (and have a blood culture obtained) in a wide variety of places, including the hospital where their CVC was inserted, another hospital, a private physician's office, or an outpatient (including emergency department) clinic, or be sent directly to a private laboratory. How can we ensure that appropriate cultures are obtained, that the specimen is processed correctly, and 
that these critical numerator data (a positive culture) get included in a database that is available to clinical and infection control personnel who are treating the patient or conducting BSI surveillance and monitoring BSI rates?

Fifth, given the above, our BSI surveillance and control efforts are seriously hampered by inadequate information systems at our healthcare facilities. To monitor our BSI rates, we need both numerator (number of BSIs) and denominator (number of CVC-days) data. Often these data are collected by infection control or other personnel who go to the microbiology laboratory and review an accession book or other paper records for the numerator data and try to identify the patients admitted or discharged from inpatient services to obtain denominator data. Outside of the intensive care unit, often the number of patient CVC-days by area or unit is not available. In the investigation by Smith et al., no routine database of the clinic patient visits by service was maintained, nor for any service was the number of patients attending the clinic, having a $\mathrm{CVC}$, or having the CVC manipulated captured for infection control or BSI rate calculation purposes. After considerable investigation of data sources and discussions with infection control, oncology, information services, and clinic personnel and through the good will of a variety of hospital personnel, the numerator and denominator data could be collected, although this required a complex system of data retrieval from several paper and computer sources and was limited to pediatric hematology-oncology patients. Perpetuation of such a system long-term, when the concern over potential increased BSI rates in this population was diminished, was unlikely. Furthermore, such a patchwork system provided no data on other at-risk populations. As the number of patients with CVCs receiving medical care in outpatient and home settings increases and the number of personnel to perform BSI surveillance is not expanded, more personnel- and time-efficient methods of capturing these data are urgently needed. One of the best options is the use of existing (or newly developed) computer information systems. Because all healthcare system encounters of any patient generate a charge, they are captured by some information system at the healthcare facility. Integrating the systems that capture the charge for insertion of the CVC into the patient during a clinic visit, the patient's CVC manipulation in the hospital, clinic, or home setting, and microbiology laboratory data, including the results of blood cultures from the patient, is the challenge for the future. Given the cost (to both the patient and the healthcare system) of BSIs, it would be very cost-effective and would greatly assist in BSI prevention to have such integrated systems. Innovative uses of integrated computer programs for monitoring CVC-associated events are beginning to emerge..$^{15}$ Finally, the events of September 11, 2001, and the following months vividly demonstrate our need for better surveillance in outpatient settings, be they emergency departments, private physician offices, clinics, or other ambulatory settings. Funding for bioterrorism to state and local health departments and to other healthcare settings may be the impetus for developing these integrated information systems.

If you think that your BSI surveillance system is capturing all of the BSIs occurring in your catchment population, perhaps you should ask the questions that Dr. Smith and her colleagues asked during their investigation and see whether you can get all of the required numerator and denominator data to calculate population-specific BSI rates. You may find that your BSI surveillance system is not as prepared to capture events that present outside the inpatient areas (clinics, emergency departments, or homes) as you think.

\section{REFERENCES}

1. Banerjee SN, Emori TG, Culver DH, et al. Secular trends in nosocomial primary bloodstream infections in the United States, 1980-1989: National Nosocomial Infections Surveillance System. Am J Med 1991;91:86S-89S.

2. Wenzel RP, Edmond MB. The impact of hospital-acquired bloodstream infections. Emerg Infect Dis 2001;7:174-177.

3. Emori TG, Edwards JR, Culver DH, et al. Accuracy of reporting nosocomial infections in intensive-care-unit patients to the National Nosocomial Infections Surveillance system: a pilot study. Infect Control Hosp Epidemiol 1998;19:308-316.

4. Smith TL, Pullen GT, Crouse V, Rosenberg J, Jarvis WR. Bloodstream infections in pediatric oncology outpatients: a new healthcare systems challenge. Infect Control Hosp Epidemiol 2002;23:239-243.

5. Manangan LP, Pearson ML, Miller E, Tokars II, Jarvis WR. Feasibility of national surveillance of healthcare-associated infections in home-care settings. Emerg Infect Dis J 2002;5:253-264.

6. Jarvis WR. Infection control and changing healthcare delivery systems. Emerg Infect Dis J 2001;7:170-173.

7. Eberhardt MS, Ingram DD, Makuc DM, et. al. Urban and Rural Chartbook: Health, United States, 2001. Hyattsville, MD: National Center for Health Statistics; 2001.

8. Kellerman S, Shay D, Howard J, et al. Bloodstream infections in home infusion patients: the influence of race and needleless intravascular access devices. J Pediatr 1996;129:711-717.

9. Fridkin SK, Pear SM, Williamson TH, Galgiani JN, Jarvis WR. The role of understaffing in central venous catheter-associated bloodstream infections. Infect Control Hosp Epidemiol 1996:17:150-158.

10. Archibald LK, Manning ML, Bell LM, Banerjee SN, Jarvis WR. Patient density, nurse-to-patient ratio and nosocomial infection risk in a pediatric cardiac intensive care unit. Pediatr Infect Dis / 1997;16:1045-1048.

11. Robert J, Fridkin SK, Blumberg HM, et al. The influence of the composition of the nurse to staffing ratio on primary bloodstream infection rates in a surgical intensive care unit. Infect Control Hosp Epidemiol 2000;21:12-18.

12. Tokars JI, Cookson ST, McArthur MA, Boyer CL, McGreer AJ, Jarvis WR. Prospective evaluation of risk factors for bloodstream infection in patients receiving home infusion therapy. Ann Intern Med 1999;131:340347.

13. Mermel LA, Farr BM, Sherertz RJ, et al. Guidelines for the management of intravascular catheter-related infections. Infect Control Hosp Epidemiol 2001;22:222-242.

14. Haley RW, Culver DH, Morgan WM, White JW, Emori TG, Hooten TM Increased recognition of infectious diseases in U.S. hospitals through increased use of diagnostic tests, 1970-1976. Am J Epidemiol 1985;121:168-181.

15. Soderberg LM. Designing and creating a central venous catheter database and electronic charting system. Journal of Intravenous Nursing 2001;24:159-168. 